\title{
Zur Frage der Ausscheidung gasförmigen Stickstoffs aus dem Thierkörper.
}

\author{
Von
}

\section{J. Seegen und J. Now a k}

(Wien.)

Wir haben in diesem Archive (Bd. XIX) Versuche iiber die Ausscheidung von gasförmigem Stickstoff aus dem Thierkörper mitgetheilt, und haben das Ergebniss dieser Versuche dahin resumirt, „jass der thierische Organismus im Stande ist, einen Theil des aus der Umsetzung der Albuminate frei werdenden Stickstoffes in Gasform auszuscheiden."

Nach Ablauf von zwei Jahren veröffentlichen M.Pette n k o fer und C. Voit eine Arbeit ${ }^{1}$ ) über unsere Versuche, die in dem Sehlusssatze gipfelt, dass das von uns erhaltene Plus an Stickstoff „das Resultat mangelhafter Beobachtung ist". Die erwähnte Arbeit ist nicht eine blosse Wortkritik, sie stützt sich anf eine stattliche Reihe von Versuchen der verschiedensten Art. Die Versuche sind nicht Respirationsversuche, welche zu einem von den unserigen entgegengesetzten Resultate fülurten, sie haben vielmehr die Aufgabe, alle möglichen Quellen, aus welchen das von uns gefundene Stickstoffplus stammen könnte, zu demonstriren, und sie sind physikalischer, chemischer und physiologiseher Natur. So vermuthen z. B. Pettenk ofer und Voit, ein Theil unseres Stickstoffes stamme aus dem durch Zersetzung des Harnstoffes stammenden Ammoniak. Sie stellen auf Grundlage dieser Vermuthung sogleich zwei Thierversuche an, es werden einmal 5 Kaninchen, und ein anderesmal ein kleiner Hund in einen Respirationsapparat gegeben und in demselben durch 72 Stunden gelassen. Sie malen den $\mathrm{Zu}$ stand des Käfigs und der Thiere nach Ablauf des Versuches mit den schwärzesten Farben. „Das Wasser rann von den Wänden auf den Boden herab, mischt sich mit Harn und Koth, und die Thiere waren besudelt von der Jauche“. Wir können versichern,

1) Zeitschrift für Biologie. XVI. Bd. 4. Heft. 
dass wir nie eine ähnliche Erfahrung über das Aussehen des Käfigs oder der Thiere machten, vielleicht weil die Ventilation eine bessere war und das exspirirte Wasser sich nicht ansammeln konnte. In dem Luftstrom, welchen P. und V. dem in so trostlosem Zastande befindlichen Athemranme entzogen, erwarteten sie grosse Mengen Ammoniak zu finden, aber die innerhalb 3 Tagen von den 5 Kaninchen exspirirte Luft enthielt nach Untersuchungen von Dr. Feder nur 0,030 grm, und die von dem kleinen Hunde exspirirte Luft nur $0,041 \mathrm{grm}$ Ammoniak. „Das entspricht so geringen Quantitäten, dass sie gegenïber den von S. und $\mathrm{N}$. erhaltenen Werthen nur in geringem Masse in Betracht kommen". Pette nkofer und $V_{0}$ it nahmen nun an, dass bei ihren Versuchen in der an Kohlensäure reichen, schlecht ventilirten Luft sich doppeltkohlensaures Ammoniak gebildet babe, welches nicht zerlegt wurde, oder dass das an den Wänden condensirte Wasser das Ammoniak resorbirt habe, dass dagegen bei der ausgiebigen Ventilation, wie sie in unseren Versuchen stattfand, ein Uebergang von Ammoniak in die Luft stattgefunden. Natürlich durfte dann auch keine Wassercondensation stattgefunden haben, und wir nehmen schon hier von diesem Zugeständnisse : Akt, um den mit Rücksicht auf das bekannte Wort Regnault's uns gemachten Vorwurf, dass es „ein barbariseher Versuch sei, drei oder vier Kaninchen in ein kleines Gehäuse zu sperren und sie darin zwei oder drei Tage lang in ihren Excrementen liegen zu lassen, ohne die gesammte Luft zu wechseln", als nicht berechtigt zu erklären. Wir haben, wie $P$. und V. zugestehen, und wie auch die Koblensäureziffern nachweisen, grïndlich ventilirt, wir hatten keine Wassercondensation an den Wänden, darum keine Schmutzjauche, wenn der Stall auch nicht beim Schlusse der Versuche allen ästhetischen Anforderungen entsprach. Es wurden nämlich die Geruchsorgane beim Oeffnen des Stalles in der unangenehmsten Weise afficirt; da wir nun diese Geriiche ,organischen Dämpfen“ zuschrieben, und in diesen auch die Ursache sahen, dass die Thiere nach längerer Zeit unwohl wurden, liessen wir bei späteren Versuchen die Luft zum Behufe der Zerstörung der organischen Substanz über glühendes Kupferoxyd streichen, was sich auch vollkommen bewährte, die Thiere blieben gesund, und beim Oeffnen des Käfigs wurde kein Sinnesorgan empfindlich verletzt. Pettenk ofer und Voit, die daran festhalten, es mitsse sich in unserem Apparate viel 
Zur Frage der Ausscheidung gasförmigen Stickstoffs aus dem Thierkörper. 385

Ammoniak entwickelt haben, und aus, diesem in irgend einer Weise das Stickstoffplus unseres Athemraumes entstanden sein, combiniren nun, die krankmachende Ursache sei nicht in organischen Dämpfen, sondern in einer Verunreinigung des Sauerstoffs zu suchen. Die Unsalubrität unseres Sauerstoffs rühre von einer Verunreinigung mit Chlor her, und dieses werde beim Leiten über gliuhendes Kupferoxyd in das wenig flüchtige Kupferchloriur umgewandelt, und darum blieben dann die Thiere gesund. P. und V. hatten nun den Gedanken, das chlorhaltige Sauerstoffgas könnte aus dem Harnstoff oder dem Ammoniak des Athemraumes Stickgas entwickeln, sie liessen Versuche nach dieser Richtnng anstellen. Dr. Feder leitete chlorhaltiges Sauerstoffgas durch eine Harnstofflösung, sie veränderte sich nicht, auch nach längerer Berührung des Gases mit Ammoniak konnte in demselben kein Stickstoff nachgewiesen werden. Dieses negative Resultat hindert aber $P$. und V. nicht, auszusprechen, es sei bei unseren Versuchen doch eine Entwicklung von Stickstoff durch das Chlor „wohl möglich, da sich dabei das im Sauerstoff befindliche Chlor allmählich im Athemraum ansammeln, und die Luft darin somit reicher an Chlor werden muisste!" - Wir besprachen zuerst jene unsern Versuchen imputirten Fehlerquellen, deren Niehtvorhandensein dureh die negativen Ergebnisse der Versuche, welche Pettenk ofer und Voit anstellten oder anstellen liessen, erwiesen wurden. Aber ausser diesen imaginären Fehlerquellen sind von $P$. und $V$. auch eine beträchtliche Zahl ganz realer Fehlerquellen nachgewiesen, von denen jede allein, wenn sie vorhanden gewesen wäre, genügt hätte, jenen Stickstoff zu liefern, welchen wir als aus dem Thierleibe stammend erklären. Die Zusammenstellung dieser bei ähnlichen Respirationsversuchen möglichen Fehlerquellen und der experimentelle Nachweis ihrer Möglichkeit ist sehr lehrreich, und die Mühe, welche P. und V. auf diese Arbeit verwendeten, ist im hohen Grade anzuerkennen, und dürfte für künftige Respirationsversuche manchen werthvollen Fingerzeig geben und manchen Irrthum verhiiten; aber die uns zunächst liegende Frage ist, haben wir diese Fehler oder einen derselben gemacht, und stammt unser Stickstoffplus aus einer dieser Quellen? Diese Frage wollen wir beantworten.

Die möglichen Fehlerquellen, welche Pettenkofer und Vọit aufführen und nachweisen, sind dreierlei Art, das Stickstoffplus kanm herbeigeführt sein 
1. durch Stickstoff, welcher sich aus dem bei der Sauerstoffbereitung verwendeten Braunstein entwickelt hat;

2. durch Stickstoff, welcher von aussen durch die das Gas absperrende Oelschicht in den Gasometer gedrungen;

3. durch ungenaue Bestimmung der Mitteltemperatur des Athemraums.

P. und V. führen die Beobachtungen mehrerer französischer Forscher an, welche nachgewiesen haben, dass der zur Sauerstoffbereitung verwendete Braunstein beträchtliche Mengen Stickstoffgas liefern kann, sie selbst baben Braunstein untersucht, welcher auf 50 grm, die mehr als 4 Liter Sauerstoff entwickeln, nur 0,4 ecm Stickstoff lieferten, es gäbe aber auch Braunsteinsorten im Handel, welche viel Stickstoff liefern.

Pettenk ofer und Voit haben ferner zahlreiche und höchst ingeniöse Versuche iiber die Diffusion von Gasen durch Oel angestellt. Die Versuche, welche sie über Diffusion von Kohlensäure durch eine Oelschicht anstellten, und die sie durch Druckdifferenz an einem Manometer bestimmten, dürften vielleicht an dem Gebrechen leiden, dass sich an dem Apparate einige Undichtigkeiten zeigten, die Luft einströmen liessen, und dass diese vielleicht auch einen Theil jener Kohlensäure aus dem Gasometer ausströmen liessen, welche als vom Oel absorbirt angegeben wurde. Für uns von Bedeutung ist jener Versuch, der über die Diffussion von $\mathrm{N}$ angestellt wurde. $P$. und $V$. liessen für diesen Versuch einen Gasometer und eine kupferne Retorte, wie wir sie benutzt hatten, eigens anfertigen, brachten an allen Verbindungsstellen Quecksilberverschlïsse an, gossen auf das Wasser im Gasometerbassin eine $4 \mathrm{~cm}$ hohe Sehicht Rüböl, entwickelten den Sauerstoff aus einem Gemenge von chlorsaurem Kali und Braunstein, der keinen Stickstoff enthielt. „Das frisch bereitete Sauerstoffgas enthielt keine merkliche Menge Stickstoff, nach 7 tägigem Stehen im Gasometer enthielt es 1,52 Vol.-Proc., nach 4 Wochen sogar 6,0 Vol.-Proc. Stickstoff". Nun berechnen sie in höchsit complicirter Weise auf Grund des von Regnault und Reiset angegebenen Verhältnisses zwischen dem verbrauchten Sanerstoff und dem ausgeathmeten Stickstoff, wie viel Sauerstoff unsere Thiere verbraucht haben können, und wie sich das Verhältniss des von uns gefundenen Stickstoffs zum verbrauchten Sauerstoff gestaltet. Das Resultat dieser Rechnung ist: „Es liesse sich die von Seegen und Nowak 
gefundene Vermehrung des Stickstoffs vollständig von einer Verunreinigung des Sauerstoffs ableiten, wenn dieser 0,75 Vol.-Proc. Stickstoff enthielte". Unsere Hunde, heisst es an einer anderen Stelle, haben durchschnittlich mehr als nochmal so viel Stickstoff ausgeathmet, als der Hund A von Regnault und Reiset, „was vielleicht davon herrührt, dass das über Wasser und Oel stehende Sauerstoffgas nochmal so viel Stickstoff aufnimmt, als das über Chlorcaleium". Der von Regnault und Reiset gefundene Stickstoff wird also nicht mehr ausschliesslich auf Undichtigkeit ihres Apparates, sondern auf Diffusion zuriickgeführt. Es gehe aus diesen Thatsachen deutlich hervor, so heisst es ferner, „dass es kein glïcklicher Gedanke von Seegen und Nowak war, Regnault's Verfahren verbessern zu wollen, indem sie wohl die Kautschukverbindungen durch Quecksilberverschlïsse ersetzten, aber das Sanerstoffgas iuber Wasser und Oel, anstatt über einer gesättigten Chlorcalciumlösung aufsammelten, sie haben durch ihre Quecksilberverschlïsse wohl einige kleine Löcher für die Diffusion verstopft, aber dafür durch die Sperrflïssigkeit ihres Gasometers ein grosses Loch aufgemacht".

„Es ist auffallend", so fahren P. und V. fort, „dass Seegen und Nowak, die von so vielem sprechen, keine Silbe dariber verlieren, wie sie die Reinheit ihres Sauerstoffgases controlirt haben".

Wir mïssen uns sehuldig bekennen, wir haben die Reinheit nicht controlirt, wir haben, wie wir dies bei Bereitung des Sauerstoffs angegeben, mehr als 100 Liter Gas ausströmen lassen, ehe wir zur definitiven Füllung des Gasometers ïbergingen. Wir haben die Bereitung begonnen unmittelbar vor dem Versuche, wir haben die Retorte so beschickt, dass während eines langen Versuches noch etwa 2-3 mal der Gasometer gefüllt werden konnte. Das verwendete Gas war also frisch bereitetes, und dieses enthält doch auch nach den Versuchen von Pettenk ofer und Voit nur minimale Mengen $N$. Regnault und Reiset benutzten frïher bereitetes, zum Zwecke der Versuche aufbewahrtes Gas, and darum mussten sie weit vorsichtiger sein in der Wahl ihrer Sperrfliussigkeit. Aber wenn auch das Loch, das wir durch die Wabl unserer Sperrflüssigkeit aufgemacht, durch die rasche Verwendung des Sauerstoffes wesentlich kleiner wurde, immerhin bleibt die berechtigte Einwendung, dass wir den Sauerstoff nicht auf seine 
Reinheit geprifft haben, und wenn selbst die Diffusion an der Verunreinigung unschädlich gewesen wäre, wie wollen wir heute beweisen, dass der von uns verwendete Braunstein stickstofffrei war, oder dass er mindestens zu der guten Sorte mit geringem Stickstoffgehalte gehört habe.

Pettenk ofer und Voit haben, nachdem sie so schweren and berechtigten Verdacht auf die Reinheit unserer Versuche, oder vielmehr auf die Reinheit des Sauerstoffs unserer Versuche gewälzt haben, in einigen markanten Worten jeden Schatten dieses Verdachts getilgt, indem sie anschliessend an die oben eitirte Frage, wie wir die Reinheit unseres Sauerstoffes controlirten, hinzufügen: „So erklärlich uns die von ihnen gefundene Stickstoffvermehrung im Athemraum bei Thieren ist, so schwer erklärlich ist es; bei der Annahme einer Verunreinigung des Sauerstoffgases, dass ihre Weingeistlampe nur eine so geringe Stickstoffzunahme geliefert hat, in dem einen Controlversuch nur 9, und in dem andern nur $88 \mathrm{mgr}$, was einem Gehalte des Sauerstoffs von nur 0,008 und 0,06 Vol-Proc. an Stickstoff entsprechen wïrde".

Bei der Annahme einer Verunreinigung des Sauerstoffs bliebe es also unerklärlich, dass bei den Verbrennungsversuchen, die 24-30 Stunden gedauert hatten, bei welchen der Sauerstoff unter ganz gleichen Bedingungen, wie bei den Thierversuchen bereitet war, dasselbe Material und dieselbe Sperrflüssigkeit verwendet wurde, doch nur sehr geringe Mengen Stickstoff vorhanden waren ; das Unerklärliche verschwindet, so wie man den bei den Thierversuchen, wie bei den Controlversuchen gefundenen Stickstoff auf seine richtige Quelle zurückführt. Die "geringe Stickstoffzunahme" der Controlversuche stammt aus den bei jeder so complicirten Arbeit unvermeidlichen Fehlerquellen, die grosse Stickstoffmenge bei den Thierversuchen wird von diesen Fehlerquellen in gleicher Weise beeinflusst, was jenseits dieser Fehlerquellen liegt, stzmmt aus dem Thierleibe. Pettenkofer und Voit haben Controlversuche stets und nachdrücklichst als Grundbedingung erklärt, wenn sie die Resultate eines Respirationsversuches gelten lassen sollten. Voit hat noch in einer seiner letzten Arbeiten ausgesprochen: „man beseitige eine so begriundete Anforderung nicht dadurch, dass man versicherte, der Apparat wäre stets in Ordnung gewesen, da man bei so complicirten Apparaten nicht immer im Stande ist vorauszusehen, ob auch alles so arbeitet, wie man voraus- 
setzt". Wir haben den Anforderungen nach Controlversuchen stets Rechnung getragen und dieselben ausgeführt, und wir können uns jetzt, nachdem uns Pettenk ofer und Voit eine Reihe neuer Fehlerquellen kennen lehrten, doppelt freuen, dass wir es gethan haben. Was hätte es uns genützt den Sauerstoff auf seine Reinheit zu prïfen, wir hätten diese Prüfung doch nur im Anfange des Versuches vornehmen können, und wenn die Prüfung auch das befriedigendste Resulltat ergeben bätte, wäre damit nicht ausgeschlossen gewesen, dass der Sauerstoff im Laufe des Versuches durch Diffusion verunreinigt gewesen wäre. Pettenkofer und Voit behaupten auf Grundlage ihrer Versuche, dass der Sauerstoff in unserer Glasglocke „nicht länger als einige Sekunden frei von Stickstoff geblieben sein kann". Unsere Controlversuche mit der Weingeistlampe sind zugleich die Controle über den Grad der Reinheit des Sauerstoffes, sie sagen aus, dass mindestens bei einem Versuche von 24-30 Stunden Dauer der Sauerstoff nur um 0,008-0,06 Vol. - Proc. verunreinigt war, diesen Controlversuchen danken wir es, dass wir auch heute, nachdem wir zwei so wichtige Fehlerquellen kennen gelernt haben, sagen können, unsere Thierversuche waren von diesen Fehlerquellen nur in sehr minimaler Weise berührt und dass Pettenk ofer und Voit dieses indirect zugestehen, indem sie auf die sonst ,unerklärliche" Verschiedenheit zwischen der bedeutenden Stickstoffvermehrung bei den Thierversuchen und der geringen Stickstoffzunahme bei den Controlversuchen nachdrïeklich hinweisen.

Aber es kann noch eine dritte Fehlerquelle vorhanden sein, durch welche der Stickstoff des Athemraums als zu gross erscheint. Diese Fehlerquelle ist die ungenaue Bestimmung der Mitteltemperatur des Athemraums, und diese Fehlerquelle habe unser Stickstoffplus mit verschuldet, da wir die Temperatur ,an dem ausgedehnten Apparate durch ein einziges an einer bestimmten Stelle des Athemraumes angebrachtes Thermometer bestimmt, und ohne weiteres vorausgesagt haben, dass die Temperatur im ganzen Raume in dem die Thiere sich befinden, sowie in der langen iibrigen Leitung absolut die gleiche sei und zwar in einem Zimmer, in welchem für gewöhnlich in der Nähe des Athemkastens und in die Leitung, durch welche die Luft eirculirt, eingeschlossen, während lange Zeit ein Verbrennungsofen glühend erhalten worden war und auch Menschen thätig waren". 
An einer andern Stelle heisst es: „eines aber ist endlich noeh in hohem Grade auffällig, das ist die fast gleiche Temperatur bei den Versuchen von Seegen und Nowak - in 34 Versuchen schwankt die Temperatur von 16.8-17.9, die grösste Schwankung beträgt also $1.1^{0}$. In manchen Versuchen, welche bis zu 110 Stunden währen, ist die Temperatur zu Anfang und zu Ende absolut die nämliche, oder sie ist nur um einige Zehntel Grade verschieden. Wenn die Arbeit von Seegen und Nowak 6 Jahre in Ansprnch genommen hat, so ist wohl ein Theil ihrer Versuche bei kälterer, ein Theil bei wärmerer Jahreszeit gemacht worden, aber die Temperatur des Raumes wird immer zu nahezu $17^{\circ}$ angegeben. Das Thermometer ist im Athemkasten aufgestellt, trotzdem ist es fast gleichgültig ob in dem Kasten ein einziges Huhn oder 5 Hïhner sich befanden, ein Kaninchen oder vier Kaninchen darin lebten. In 18 Versuchen brannte ein Verbrennungsofen im Zimmer nahe dem Athemraum, die Temperatur wurde dadurch nicht beeinflusst. Es ist uns absolut unmöglich dieses merkwürdige Gleichbleiben der Temperatur zu verstehen. Wir glauben, dass die Annahme der gleichen Temperatur an allen Stellen des Athemraumes, und in der langen Röhrenleitung, sowie auch die geringen Schwankungen der Temperatur bei den verschiedenen Versuchen zu so gewichtigen Bedenken gegen die Versuchsanordnung von Seegen und Nowak Veranlassung geben, dass wir vor deren Hebung die dadurch erhaltenen Resultate nicht als richtig ansehen können".

In diesem Schlusssatze, in welchem Pettenkofer und Voit ihren Bedenken gegen unsere Versuche Ausdruck geben, sind weit auseinander liegende Dinge zusammen geworfen, unsere "Annahme" der gleichen Temperatur an allen Stellen des Athemraumes und der langen Röhrenleitung auf Grundlage der Angabe eines Thermometers kann richtig oder unrichtig sein, darüber wollen wir später sprechen, aber die geringeren Schwankungen in der Temperatur bei den' verschiedenen Versuchen beruhen nicht auf einer Annahme, sie sind einfach eine Thatsache, und zwar eine Thatsache, die durch ein sehr einfaches und sehr verlässliches Instrument, durch ein gutes Thermometer, constatirt ist, zum richtigen Ablesen braucht es nur ein gesundes Auge und zum richtigen Registriren nur - Ebrlichkeit. Wir wollen gerne annehmen, dass unsere loyalen Gegner die Tragweite ihres "Bedenkens" nicht bedacht haben. Zur Erklärung dieses „merkwürdigen 
Gleichbleibens" wollen wir hinzufügen, dass unsere Versuche sich über einen Zeitraum von 6 Jahren erstrecken, aber nu r im Winter ausgeführt wurden, und die Temperatur eines Zimmers im Winter kann man, wie dies jeder wohl geordnete Haushalt beweist, in engen Grenzen sehwankend erhalten. Der Verbrennungsofen wurde natürlich viele Stunden vor dem Schlusse des Versuches ausser Thätigkeit gesetzt, und darum war die Schlusstemperatur nicht dadurch beeinflusst, diese Lösung für das merkwürdige Verhalten konnten Pettenkofer and Voit leicht finden, nachdem sie sich durch einen directen Versuch iiberzeugt hatten, dass schon 52 Minuten nach dem Abkühlen des Verbrennungsofens die Zimmertemperatur von $22^{\circ}$ auf $19.4^{\circ}$ gesunken war und dass nach dieser Zeit die Temperatur im Innern nur "etwas höher war als aussen"; wenn sie ihren Thermometer eine Stunde später angesehen hätten, wäre wohl die urspriungliche Zimmertemperatur von $17.7^{\circ}$ innen und aussen vorhanden gewesen. Der Thatsache, dass die Temperatur im Athemraume nur um Weniges sehwankt, ob ein einziges Huhn oder fünf Hühner, ein Kaninchen oder drei Kaninchen sich in demselben befinden, können wir noch eine andere, eben so frappirende, an die Seite stellen. In unserem Versuch VII, mit 2 Hühnern angestellt, mit der Anfangstemperatur von 16.9, war die Temperatur des Athemraumes nach 9 Stunden 17.5 und nach 72 Stunden 17.4 ! Innerbalb dieser 63 Stunden hatten doch die Thiere gewiss eine erkleckliche Menge Wärme producirt und doch war die Temperatur des Athemraumes nicht gestiegen. Es ist also einfach diese Wärme nach aussen geströmt, die Temperatur der Luftschichte im Innern des Athemraumes bat sich durch den diunnen gut leitenden Blechkasten mit der Temperatur der äusseren Luft in's Gleichgewicht gesetzt, nnd darum konnte sich die Differenz in der Wärmeproduction von einem oder drei Kaninchen von einem oder fünf Hühnern nicht in auffallender Weise manifestiren. Pettenk ofer und Voit haben ja selbst in einem ad hoc angestellten Versuche es erfahren, dass die Temperatur eines Athemkastens in welchem Weingeist gebrannt hatte, nach dem Auslöschen der Flamme innerhalb 37 Minuten von $27^{\circ}$ bis auf die Zimmertemperatur von $17^{\circ}$ gesunken war. Dieses sind die $\mathrm{Er}$ klärungen für die von uns registrirten Thermometerbeobachtungen.

Ein ernstes and berechtigtes Bedenken ist es, ob die Luft im 
Athemkasten so gemiseht war, dass man im Stande ist, aus der Temperaturanzeige eines im Athemraume anfgestellten Thermometers auf die Temperatur der gesammten Luft des Athmungsraumes und der Leitungsröhren zu schliessen. Wir gestehen, in der langen Abhandlung von Pettenk ofer und Voit war dieses Bedenken das Einzige, das uns imponirte. Wir hatten, als wir unsere Versuche anstellten, wohl daran gedacht, ob wir den Apparat ins Wasser stellen sollten, es hätte olne Zweifel einen Werth, wenn man den gesammten Apparat mit allen Leitungsröhren mit Wasser hätte umgeben können, es hätte dadurch mit grösserer Bestimmtheit eine Gleichheit in der Temperatur des Athemraumes und der Leitungsröhren herbeigefuihrt werden können, während es auf der Hand lag; dass vielleicht an einzelnen Stellen der Leitungsröhren, speciell in der Nähe der Absorptionsgefässe eine etwas höhere Temperatur sein könnte, aber der ganze Apparat konnte aus technischen Gründen nicht von Wasser umgeben werden, und so mussten wir uns dabei beruhigen, dass jene Partien der Leitung, in welchen durch Absorption eine höhere Temperatur entsteht, doch nur einen kleinen Bruchtheil des Apparates ausmachen, dass sich endlich diese kleine Temperaturdifferenz auch schon bei der Anfangsanalyse, die doch gewöhnlich eine Stunde nach Beginn des Versuches gemacht wurde, wenigstens theilweise geltend mache. Den Athemraum ins Wasser zu stellen, wie Regnault und Reiset, und wie wir es bei früheren Versuchen gethan, hat keinen besonderen Werth, wenn man nicht darauf hält, dass Anfangs- und Endtemperatur gleich seien. Für die Gleichmässigkeit der Temperatur im Athemraum im Moment des Ablesens hat es keine Bedeutung, ob das umgebende Medium Luft oder Wasser ist. Regnault und Reiset glaubten an die Gleichmässigkeit dieser Temperatur im Athemraum, denn sie bestimmten sogar dieselbe durch ein einziges in dem umgebenden Wasser befindliches Thermometer, wir konnten um so mehr hoffen, dass die Temperatur durch ein im Athemraume angebrachtes Thermometer richtig angezeigt werden würde. Sollte nun wirklich das fortgesetzte Pumpen nicht genügen, die Luft zu mischen und eine gleichmässige Temperatur herbeizuführen? Wir wollten, nachdem P. und $V$. diese Zweifel wach gerufen, dieselben durch einen Versuch lösen. Wir setzten in unsern 70 Liter fassenden, mit der Glasglocke geschlossenen, Athemkasten zwei Kaninchen. In den 
Athemraum wurden 3 Thermometer gesteckt, der eine der Decke der Glasglocke nahe, der andere reichte an den Boden, der dritte in die Mitte des Athemraumes, die Luft des Saales hatte $16.8^{\circ}$. Der Versuch begann am $3 / 4$ um $1 / 211$ Uhr:

$\begin{array}{ccc}\text { 1/211 Uhr } & 1 / 212 \text { Uhr } & 4 \text { Uhr } \\ \text { ob. Th. } 17.3^{\circ} & 17.5 & 18 \\ \text { m. Th. } 17.0 & 17.2 & 17 \\ \text { unt. Th. } 17.0 & 17.2 & 17\end{array}$

Nun begannen wir zu ventiliren, etwa 500 Liter in der Stunde, es zeigten nun die Thermometer:

$\begin{array}{lrr} & 1 / 25 & \text { um } 5 \\ \text { ob. } & \text { Th. } 17.3 & 17.1 \\ \text { m. } & \text { Th. } 16.7 & 16.9 \\ \text { unt. } & \text { Th. } 16.7 & 16.9\end{array}$

Nachdem wir also e ine Stunde ventilirt hatten, betrug die Temperaturdifferenz 0,2 und zwar bei einer Ventilation die nur $1 / 3$ derjenigen betrug, die wir in unseren Versuchen angewendet hatten. Wir konnten also darüber beruhigt sein, die Temperatur unseres Athemraumes warde durch unser Thermometer correct angezeigt. Voit und Pettenkofer theilen zwei, Versuche, mit 3 Kaninchen, mit, die sie in einem Athemkasten anstellten, welcher einen Kubus von je $73 \mathrm{~cm}$. Seitenlänge darstellt, also 389 Liter fasst und in welchem 12 Thermometer aufgestellt waren. Bei einer Zimmertemperatur von 8.2-8.70 danerte es 25 Minuten bis die Temperatur gleich blieb, die Temperatur schwankte von 8.5-11.9. Von einem zweiten Versuche welcher in Bezug auf Zimmertemperatur dem unsrigen nahe stand, die Zimmertemperatur war 19.2, die Ventilation 666 Liter in der Stunde, heisst es: „Hier nimmt an einigen Stellen nach 15 Minuten die Temperatur nicht mehr zu, an andern währt es 35 Minuten bis eine Constanz"eingetreten ist. Die Temperaturdifferenzen betragen $1.6^{\circ}$." Es ist nicht deutlich gesagt, ob diese Temperaturdifferenzen vorhanden waren, ehe die Temperatur constant war, oder ob sie noch bestand, als die Temperatur stetig geworden war. Aber wenn auch letzteres der Fall wäre, ist zu bemerken, dass der von $P$. und V. verwendete Athemkasten einen Kubus repräsentirte, also wahrscheinlich viele todte Winkel hatte, während der unserige rund war, also weit besser ventilirt werden konnte, es ist ferner nicht gesagt aus welchem Material ihr Kasten war, der unserige war aus dïnnem Kupferblech, der Athemraum 
ihres Kastens betrug 389 Liter, wir haben nur 1 oder 2 mal einen Kasten von 310 Liter angewendet, für alle uibrigen Versuche dienten Kasten von 50-70 Liter, endlich dauerte die Ventilation nur 35 Minuten, sie wären bei fortgesetzter Ventilation wohl zu demselben Resultate gelangt, zu dem wir gelangt sind. In einem von ihnen angeführten Versuche mit einer brennenden Weingeistlampe schwankte die Temperatur im Innern zwischen 17.5-270, die äussere Temperatur betrug 17.10. Nach, dem Auslöschen der Flamme und fortdauernder Ventilation, währte es mindestens 37 Minuten bis die Abkühlung bis zur Temperatur der Zimmerluft an einigen Thermometern eingetreten war, an andern war sie noch nach 67 Minuten nicht vollendet". Offenbar war nach dieser Zeit an keinem Thermometer ein bemerkenswerther Unterschied gegen die äussere Temperatur nachzuweisen, da sonst die Zifferdifferenz statt des vagen Ausdrucks ,nicht vollendet" angeführt worden wäre. Also auch bei einer so mächtigen Wärmequelle, wie es eine brennende Weingeistlampe ist, genügte eine Ventilation von etwas über einer Stunde um die Luft zu mischen, und die Temperatur mit der änssern mindestens nahezu gleich zu machen. Dass wir es mit dem Auspumpen ernst genommen haben, anerkennen P. u. V. für unsere Controlversuche, es wäre ihnen sogar sehr interessant zu wissen, wie wir auf den guten Gedanken gekommen sind, nach dem Erlöschen noch zu ventiliren und ,wenn das nachträgliche Auspumpen für die Versuche mit dem Weingeist nöthig war, so musste es auch bei den Thierversuchen geschehen". Diesen letzt eitirten Satz können wir nur als einen Lapsus calami ansehen. Der Weingeistversuch ist zu Ende, wenn der Weingeist ausgebrannt ist, wenn die Lampe erliseht; ohne an der Versuchsanordnung etwas zu ändern, kann man noch, so lange man will fortventiliren, d. h. die Luft des Apparates mischen. Der Thierversuch ist abgeschlossen, wenn man das Thier aus dem Athemraum entfernt, wann soll das "nachträgliche Auspumpen" stattfinden? P. and V. meinten vielleicht, der Thierversuch, also auch die Ventilation hätte fortgesetzt werden müssen, nachdem die künstliche Wärmequelle, der Verbrennungsofen ausser Thätigkeit gesetzt war. Dieser Anforderung haben wir selbstrerständlich Geniige geleistet, es hat uns dabei derselbe ,gute Gedanke" geleitet, der uns bei den Controlversuchen leitete, die Luft des Athemraumes wie die des Verbrennungsraumes möglichst gut $\mathrm{zu}$ mischen und 
diese Mischung so lange fortzusetzen, bis die äussere und die innere Temperatur sich möglichst ins Gleichgewicht gesetzt hatten. Die Temperaturen, die wir beim Abschluss unserer mit dem Verbrennungsofen ausgefuhrten Versuche gefunden und registrirt haben, sind wohl die besten Belege, dass wir dieser Anforderung nachgekommen sind.

Schliesslich wird noch bemerkt, dass alle Leitungsröhren in unserem Apparate und ebenso das Thermometer "an der Decke" angebracht sind, und "es ist denkbar, dass dadurch keine vollständige Mischung zu Stande kommt und dass eine Luft zur Untersuchung kommt, welche nicht die mittlere Zusammensetzung und Temperatur der Luft im Kasten besitzt". Wir möchten gegen diesen Einwurf bemerken, 1) dass die Luft im oberen Theile des Athemraumes, wenn sie nicht gehörig gemischt gewesen wäre, eine höhere Temperatur gehabt hätte, und indem wir diese Luft zur Untersuchung nahmen, konnte der Fehler nur zu unseren Ungunsten ausfallen, die ticfere und dichtere Luft hätte noch mehr Stickstoff enthalten; 2) derselbe Apparat mit den oben ausmündenden Röhren und dem oben angebrachten Thermometer wurden auch für die Controlversuche gebraucht, und müssten diese auch alle die Fehler nachweisen, welche aus dieser vermeintlichen unvollkommenen Luftmischung hervorgehen.

Die Athmungsversuche sind unendlich schwierig, in diesem Punkte stimmen wir mit Pettenk ofer und Voit vollkommen überein, und wir wissen es am besten, dass wir noch nicht an der Grenze der möglichen Genauigkeit angekommen sind, aber alle die Fehlerquellen, die auf unsere Versuche störend einwirkten, oder einwirken konnten, Stickstoffgehalt des Braunsteins, Stickstoffeintritt durch Diffusion, unvollständige Mischung der Luft des Athemraumes mit der der Leitungsröhre, sie waren in ganz gleichem Maasse bei den Controlversuchen wie bei den Thierversuchen vorhanden, und das in den Controlversuchen gefundene Stickstoffplus ist das Maass für diese Fehlerquellen, was bei den Thierversuchen jenseits dieses aus den Fehlerquellen stammenden Stickstoffplus liegt, stammt aus dem Thierleibe, ist aus der Umsetzung der Eiweisskörper entstandenes und ausgeathmetes Gas.

Pettenkofer und Voit schliessen die Kritik unserer Versuche damit, es sei nach den Versuchen von M. Gruber unmög. 
lich, dass aus der Zersetzung des Eiweisses herrührendes Stickgas den Körper verlasse, denn er erhielt in einem Versuche von 17 Tagen bei Einnahme von $368.53 \mathrm{gr} \mathrm{N}$ in Ham und Koth $368.28 \mathrm{~N}$, während Seegen und Nowak behaupten, dass $9.5 \%$ des umgesetzten Stickstoffes vom Körper in Gasform weggehen, wonach im Harn und Koth nur $333.52 \mathrm{gr} \mathrm{N}$ bätten gefunden werden mïssen.

Wir möchten iiber die Beweiskraft des Gruber'sehen Versuches nur einige Worte hinzufügen. In den ersten Sätzen, mit welehen Pettenk ofer und Voit ihre Kritik unserer Respirationsversuche einleiten, sagen sie, ein indirecter Versuch, d. h. ein solcher, bei welchem nur Harn und Koth untersucht wird, könne nur dann Beweiskraft haben, „wenn das Thier weder Stickstoff von seinem Körper verliert, noch Stickstoff an seinem Leibe ansetzt", d. h. mit andern Worten, wenn Stickstoffeinnahme und Ausgabe sich decken, bei vollkommen gleich bleibendem Körpergewichte. Gruber's Versuch entspricht dieser Anforderung nicht, sein Thier hat um $940 \mathrm{grm}$ an Körpergewicht abgenommen. Er will nach einer frühern, in den obigen Worten Pettenkofer's und Voit's verurtheilten Praxis, diesen Verlust auf Wasser oder Fett beziehen. Wie natürlich, ist dies nie zu beweisen. Wenn diese $940 \mathrm{grm}$, die das Thier verloren hat, umgesetztes Fleisch sind, steht der Stickstoffausfuhr von 368 grm eine Einfuhr von $368+34.8 \mathrm{grm}$ gegenüber, diese $34.8 \mathrm{grm}$ sind in den Excreten nicht nachgewiesen, sie können also in der exspirirten Luft vorhanden gewesen sein.

Gruber's Versuch unterscheidet sich von den Voit'schen Versuchen dadurch, dass er für den Stickstoffgehalt des Fleisches nicht eine Mittelzahl annahm, sondern die Stickstoffmenge des verbranchten Fleisches direct bestimmte. Er bemüht sich zu beweisen, dass der Unterschied dieser beiden Methoden nur ein sehr geringer sei. Eine einfache Rechnung beweist, dass diese Anschanung nicht ganz richtig ist. In der ersten 7tägigen Fïtterungsperiode, in welcher Gruber den Ngehalt des Fleisches bestimmte, betrug dieser $3.68 \%$. Das Thier erhielt in diesen 7 Tagen $154.8 \% \mathrm{~N}$. Würde die Voit'sche Stickstoffmittelziffer der Einnahme zu Grunde gelegt worden sein, dann hätte die Einnahme per Tag $6 \times 3.4=20.4 \mathrm{grm}$ und fïr 7 Tage 142.8 betragen, was eine Differenz ro n circa $8 \%$ beträgt. Gruber selbst hat also durch seinen Versuch bewiesen, dass bei allen Versuchen, die mit Zugrundelegung der Voit'schen Stick- 
stoffmittelzahl ein Gleichgewicht zwischen Einnahme und Ausgabe nachweisen, ein Deficit v on $8 \% \mathrm{~m} \ddot{\mathrm{g} l i c h}$ ist, welches in anderer Weise als durch Harn und Koth den Körper verlassen haben kann.

Dazn möchten wir noch bemerken, dass wir die von Gruber gefundenen Stickstoffzahlen des Fleisches nicht für richtig anzunehmen im Stande sind, weil er sowohl nach Will Varrentrapp wie nach Dumas die gleiche Ziffer erhalten hat, was dem Resultate unserer Untersuchungen sowohl jener die wir gemeinschaftlich ausführten, wie jener, die früher der eine von uns $(\mathrm{N})$ ansgeführt hat, entsehieden zuwider läuft. Gruber sagt nun zwar, unsere Analysen seien fehlerhaft ,und verdienen keine Beachtung.", aber er hat es unterlassen, den Fehler nachzuweisen. Dass wir versäumt hätten, das zu analysirende Fleisch fein zu pulvern, kann wohl nicht ernst genommen sein, diese Vorsichtsmassregel gehört zu den Elementen einer sorgfältigen Analyse. Gruber behauptet ferner, es sei vergeblich, uns Fehler nachzuweisen, da wir alle uns gegen die Ausführung der Methoden gemachten Einwendungen zurïckweisen und er citirt als Stiitze unsere Arbeit im Archiv für die ges. Phys. Bd. IX. S. 227. Gruber scheint vergessen zu haben, dass wir in dieser Arbeit nicht die gemachten Einwendungen von Maerker, Kreusler und Ritthausen zurïckgewiesen haben, sondern uns bemüht haben, sie zu widerlegen, und dass gerade auf Grundlage dieser Widerlegung einige unserer früheren Gegner und speciell Ritthausen, den er mit Recht als höchste Autorität auf dem Gebiete der Eiweissanalysen anerkennt, ihre Analysen controlirt haben und dass Ritthausen dann. zu dem Resultate gelangte, dass für Eiweisskörper „die Verbrennung mit Natronkalk unvollständig sei, dass sie ungenaue zu niedrige Zahlen für den Gehalt an $\mathrm{N}$ liefert". Wie Gruber selbst anfuihrt, hat ihn Dr. Feder darauf aufmerksam gemacht, dass auch beim Pepton die beiden Methoden grosse Differenzen in der Stickstoffzahl geben, und es ist dies eine weitere Bestätigung dafür, dass für Eiweisskörper die Will-Varrentrapp'sche Methode nicht ausreicht. Gruber wird doch noch abwarten miussen, dass seine Fleischanalysen von ebenso competenten Seiten bestätigt werden, wie unsere Eiweissanlysen bestätigt worden, ehe er berechtigt sein wird, unsere Fleischanalysen als nicht beachtungswerth zu betrachten.

Wir wollen in die Details der „Gruber'schen Arbeit nicht 
Seegen u. Nowak: Zur Frage der Ausscheidung gasförmigen Stickstoffs.

weiter eingehen ${ }^{1}$ ). Gruber's Versuche schliessen sich an die zahlreichen Versuche, die ursprünglich von $B$ ischoff und $V_{0}$ it, später von Voit allein und nach ihm von seinen Schülern an landwirthschaftlichen Stationen ausgeführt wurden, um den Lehrsatz zu stiitzen, dass aller umgesetzte Stickstoff im Harne erscheine und dass kein anderer Abzugsweg für denselben existire. Wir haben den sehr schwierigen Weg der Respirationsversuche betreten, und diese Versuche haben uns, wie schon früher Regna ult und Reiset den Beweis geliefert, dass ein Theil des im Körper aus der Umsetzung der Albuminate entstandenen Stickstoffs in der Exspirationsluft vorhanden sei. Wir haben dureh diesen directen Nachweis des Stickstoffs uns die Berechtigung verschafft, die indirecten Beweise für das Nichtvorhandensein des Stickstoffes in der exspirirten Luft nicht für vollgültig anzusehen. Pettenk of e r und Vo it haben, da sie unsere Versuche nicht wiederholen wollten, den einzig richtigen Weg eingeschlagen, die Beweiskraft unseres ad oculos demonstrirten Stickstofís als hinfällig darzustellen, indem sie denselben als ans Fehlerquellen stammend erklärten. $O b$ ibre Erklärung richtig ist, wird jeder, dem es in dieser hochwichtigen Frage um die Wahrheit zu thun ist, aus unserer Analyse ihrer Kritik zu beurtheilen im Stande sein.

1) Auch der gegen uns angeschlagene Ton soll unberücksichtigt bleiben. Gegenüber der kleinen Freundlichkeit M. Gruber's bei der meinen Namen führenden Methode der Stickstoffbestimmung Schneider's Namen zu substituiren, möchte ich nur erwähnen, dass, als ich vor 17 Jahren nach langen vergleichenden Versuchen diese Metbode einführte, es mit den Worten geschah: „Die Stickstoffbestimmung geschah nach Voit's Methode durch Glühen mit Natronkalk in einem von mir mit Hülfe von Prof. Schmeider modificirten Apparate." (Sitzungsberichte der kais. Akademie der Wissenschaften. 1864). 